\title{
Severe or lethal toxicities with nucleosidic analogs: time for action
}

\section{"Owing to the number of patients with cancer likely to receive these drugs as part of their treatment, implementing routine CDA testing as a means to limit drug-induced toxicities sounds like a reasonable option to ensure better safety when handling nucleosidic analogs."}

KEYWORDS: capecitabine $\approx$ CDA $\approx$ cytidine deaminase $\approx$ gemcitabine $\approx$ nucleosidic analogs $=$ pharmacogenetics $\|$ toxicity

\section{Nucleosidic analogs in the era of pharmacogenetics}

Nucleosidic analogs are mainstays for treating several hematological malignant disorders and solid tumors, both in children and in adults. As for most cytotoxics, the therapeutic window of nucleosidic analogs is narrow and interindividual variability in their pharmacokinetic profiles may lead to life-threatening toxicities or treatment failure. Identifying genetic markers associated with response or toxicities with anticancer drugs is a major trend in clinical oncology. Beside molecular determinants at the tumor level, constitutional genetics can help to forecast clinical outcome related to changes in drug-metabolizing enzymes of anticancer agents. Changes in pharmacokinetics and subsequently in drug exposure are often driven by genetic polymorphisms affecting metabolizing enzymes in the liver, such as the canonical DPD-deficiency syndrome in patients administered 5-fluorouracil [1].

\section{"The drawback of this single key enzymatic catabolic pathway is that subjects harboring CDA deficiency are at risk for drug overexposure with severe adverse reactions."}

Disposition of nucleosidic analogs such as gemcitabine, cytarabine, azacytidine or capecitabine is primarily dependent upon a liver catabolic or anabolic biotransformation by CDA, a ubiquitous enzyme affected by several genetic polymorphisms [2]. Toxicity of nucleosidic analogs is usually moderate when compared with other anticancer drugs; despite this, some patients display unpredictable adverse events revealing the possibility of a specific pharmacogenetic syndrome that needs to be addressed [3-5].

\section{Integrating pharmacogenetics into gemcitabine dosing}

Gemcitabine $\left(2^{\prime}, 2^{\prime}\right.$-difluorodeoxycytidine) is a major anticancer drug acting as an antimetabolite with a growing use in solid tumors such as lung, pancreatic or gynecological cancer as part of various innovative chemotherapy protocols (e.g., Gemox and Gemcap regimens). Gemcitabine exerts its antiproliferative activity after cellular uptake and nucleosidic phosphorylation, yielding active metabolites responsible for DNA synthesis inhibition. The catabolic pathway of gemcitabine depends upon a deamination step performed by CDA leading to the inactive metabolite $2^{\prime}, 2^{\prime}$-difluorodeoxyuridine. The vast majority of the administered drug dose $(\sim 80 \%)$ is inactivated through this limiting step and secondarily recovered as $2^{\prime}, 2^{\prime}$-difluorodeoxyuridine in urine. The drawback of this single key enzymatic catabolic pathway is that subjects harboring CDA deficiency are at risk for drug overexposure with severe adverse reactions. Recently, several studies have focused on severe toxicities in patients treated with gemcitabine [3-5].

It has been hypothesized that impaired liver detoxification could explain the occurrence of life-threatening toxicities in patients with CDA deficiency. Approximately 25 SNPs were reported in the Single Nucleotide Polymorphism Database (dbSNP) to occur in the coding sequence of $C D A$, and more than 650 SNPs in the gene region. Although the genotype-tophenotype correlation of CDA is not yet fully elucidated, several polymorphisms (e.g., 79A $>\mathrm{C}$, $208 \mathrm{G}>\mathrm{A}$, -del31C and $435 \mathrm{~T}>\mathrm{C}$, to name but a few) have been identified and sometimes associated with higher incidence of severe toxicities in patients upon gemcitabine intake $[4,6-8]$.
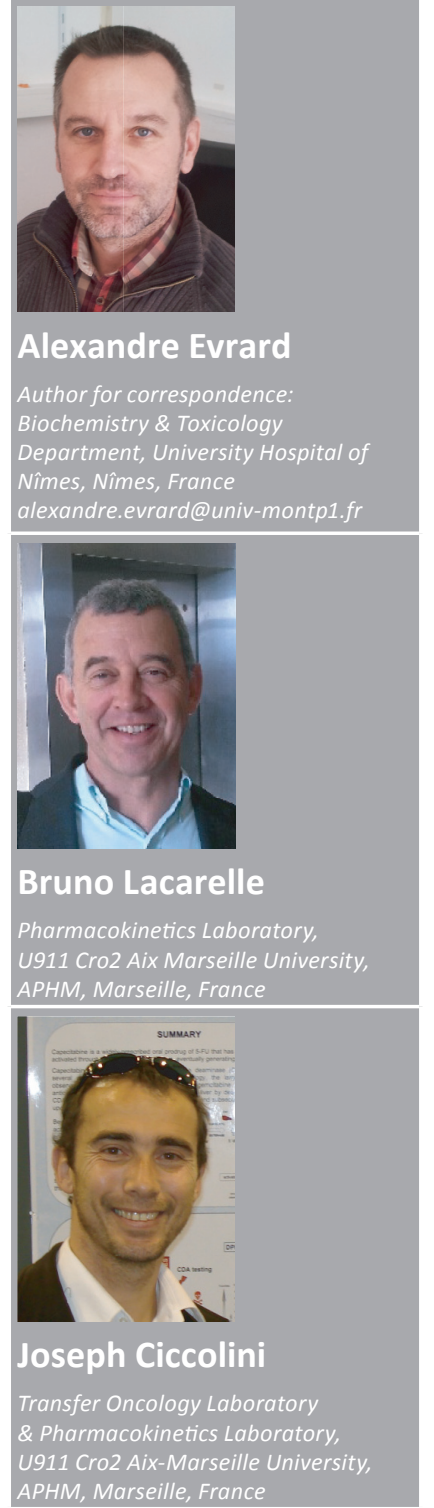

Future $\mathrm{fSS}$ 
Alternatively, the quantification of CDA activity in serum based on a spectrophotometric assay as a surrogate marker was proposed as a standard pretreatment screening $[9,10]$. The $208 \mathrm{G}>\mathrm{A}$ SNP has been first evidenced in a Japanese patient showing extremely severe toxicities upon gemcitabine administration [4]. Another case report demonstrated that CDA deficiency in relation to the c. $246+37 \mathrm{G}>\mathrm{A}$ mutation could be the cause of a case of toxic death in a patient treated with the gemcitabine-carboplatin combination [11]. Another study has concluded that the $79 \mathrm{~A}>\mathrm{C}$ polymorphism was predictive of both toxicity and efficacy in patients treated with gemcitabine-based therapy for lung cancer [12] .

The role that CDA deficiency could play in gemcitabine use has been further evidenced in another study showing that CDA activity in serum below $1.4 \mathrm{U} / \mathrm{mg}$, a value found in $10-15 \%$ of patients, was associated with the risk for developing early severe toxicities in any gemcitabine-containing regimen [9].

\section{"Patients experienced severe and eventually unrecoverable toxicities after being administered standard [capecitabine] dosage."}

Overall and despite remaining issues with understanding genotype-phenotype relationships with CDA, there is a rising number of clinical evidence strongly suggesting that gemcitabine administration could be rendered safer by sorting patients according to their CDA status prior to starting the treatment. Beside the issue of preventing gemcitabine-related toxicities in patients with CDA deficiency, a clinical report has shown that ultrarapid-metabolizer 'rapid-deaminator' patients, a status found in approximately $20 \%$ of the population, could conversely be at risk of treatment failure with gemcitabine, as suggested by a preliminary report conducted in patients with pancreatic cancer [13].

The rising concern regarding dysregulation affecting CDA in gemcitabine-treated patients has prompted several groups to further study the role this polymorphic enzyme could play in the clinical outcome with other key nucleosidic analogs that are substrates for CDA too $[14,15]$. Some recent clinical reports have suggested that CDA deficiency could be at the origin of a severe toxicity in a young patient treated with a cytarabine-based regimen [16], and could also be linked to lethal toxicities with azacytidine [17].

\section{Incidence of CDA ultrarapid- metabolizer status in capecitabine- based chemotherapy}

Capecitabine is an oral prodrug following a three-step biotransformation pathway at the liver and tumor levels by successive action of carboxyl esterase, CDA and thymidine phosphorylase to yield 5-fluorouracil. Conversely to gemcitabine, $\mathrm{CDA}$ is implicated in the activation pattern of oral capecitabine, and patients with ultrarapidmetabolizer status could thus be at risk of overactivating the prodrug to 5-fluorouracil, eventually leading to severe toxicities. This has been first evidenced in a couple of patients undergoing capecitabine-based therapy $[18,19]$. Patients experienced severe and eventually unrecoverable toxicities after being administered standard dosage. In both cases, CDA activity proved to be markedly higher as compared with other patients, thus supporting the hypothesis that ultrarapid deamination could explain the cases.

Caronia et al. investigated the relevance of various polymorphisms affecting key enzymes of the capecitabine metabolism pathway as putative prognostic markers for treatment-related severe toxicities. This candidate-gene approach concluded that severe hand-foot syndrome upon capecitabine intake could be attributed to the rs3215400 SNP across the $C D A$ gene promoter (-31delC). The $\mathrm{C}$ insertion was found to be associated with enhanced transcription by creating a binding site for the transcriptional regulator E2F [19]. Extensive phenotype in patients bearing this polymorphism could explain the higher incidence of hand and foot syndrome, although no data on enzymatic activities were made available.

At our institutes, in 2009 we demonstrated the first severe toxicity case upon capecitabine intake in a female patient with such an extensive phenotype for CDA. Although no polymorphism was identified, functional screening for CDA activity demonstrated that this patient displayed an enzymatic activity $180 \%$ higher than the mean values usually recorded in cancer patients, thus demonstrating that extensive CDA could be a condition associated with severe toxicities in capecitabine-treated patients [18]. Although there is no doubt about the existence of ultrarapid-metabolizer patients harboring enhanced CDA activity, the genetic basis for this abnormality remains unclear.

Indeed, the allelic frequency of rs3215400 is high ( 0.564 vs 0.436 for the $C$ allele) suggesting that this SNP alone does not systematically lead to CDA overexpression, a phenotype observed 
in less than $20 \%$ of our patients $[9,13]$. This suggests that no single SNP entirely accounts for observed variation in CDA activity in patients and that haplotype analysis might be more powerful to predict CDA transcription, as suggested previously in functional studies [20].

\section{Future perspective}

Despite conflicting genotype-to-phenotype relationships, a variety of clinical reports have demonstrated, either on a genotypic or a phenotypic basis, that deregulated CDA could be a culprit for severe and sometimes lethal toxicities with nucleosidic analogs. Overall, CDA status seems to be a critical condition associated with the clinical outcome of major anticancer agents such as gemcitabine and capecitabine. Although smaller to date, clinical evidence exists regarding a possible impact with cytarabine and azacytidine too. Owing to the number of patients with cancer likely to receive these drugs as part of their treatment, implementing routine CDA testing as a means to limit drug-induced toxicities sounds like a reasonable option to ensure better safety when handling nucleosidic analogs. Many genetic and epigenetic events on $C D A$ genes are likely to interfere with the genotypeto-phenotype relationships, as some of these regulations have yet to be discovered. However, we do think that the time for action has come, since the quantification of CDA activity in serum could be easily used as a surrogate marker, as several phenotype-based methods have been made available in the literature. Larger prospective clinical trials are currently being undertaken to untangle the role of genetic variations in CDA deficiency. These studies should also address the question of the right dose since the next step forward after identification of patients at risk will be dosing guidance and personalized prescription.

\section{Financial \& competing interests disclosure}

The authors have no relevant affiliations or financial involvement with any organization or entity with a financial interest in or financial conflict with the subject matter or materials discussed in the manuscript. This includes employment, consultancies, honoraria, stock ownership or options, expert testimony, grants or patents received or pending, or royalties.

No writing assistance was utilized in the production of this manuscript.

\section{References}

1 Ciccolini J, Gross E, Dahan L, Lacarelle B, Mercier C. Routine dihydropyrimidine dehydrogenase testing for anticipating 5-fluorouracil-related severe toxicities: hype or hope? Clin. Colorectal Cancer 9(4), 224-228 (2010).

2 Gilbert JA, Salavaggione OE, Ji Y et al. Gemcitabine pharmacogenomics: cytidine deaminase and deoxycytidylate deaminase gene resequencing and functional genomics. Clin. Cancer Res. 12(6), 1794-1803 (2006).

3 Gupta N, Ahmed I, Steinberg H, Patel D, Nissel-Horowitz S, Mehrotra B. Gemcitabineinduced pulmonary toxicity: case report and review of the literature. Am. J. Clin. Oncol. 25(1), 96-100 (2002).

4 Yonemori K, Ueno H, Okusaka T et al. Severe drug toxicity associated with a single-nucleotide polymorphism of the cytidine deaminase gene in a Japanese cancer patient treated with gemcitabine plus cisplatin. Clin. Cancer Res. 11(7), 2620-2624 (2005).

5 Mercier C, Raynal C, Dahan L et al. Toxic death case in a patient undergoing gemcitabinebased chemotherapy in relation with cytidine deaminase downregulation. Pharmacogenet. Genomics 17(10), 841-844 (2007).

6 Sugiyama E, Kaniwa N, Kim SR et al. Pharmacokinetics of gemcitabine in Japanese cancer patients: the impact of a cytidine deaminase polymorphism. J. Clin. Oncol. 25(1), 32-42 (2007).

7 Maring JG, Wachters FM, Slijfer M et al. Pharmacokinetics of gemcitabine in nonsmall-cell lung cancer patients: impact of the $79 \mathrm{~A}>\mathrm{C}$ cytidine deaminase polymorphism. Eur. J. Clin. Pharmacol. 66(6), 611-617 (2010).

8 Farrell JJ, Bae K, Wong J, Guha C, Dicker AP, Elsaleh H. Cytidine deaminase singlenucleotide polymorphism is predictive of toxicity from gemcitabine in patients with pancreatic cancer: RTOG 9704.

Pharmacogenomics J. 12(5), 395-403 (2012).

9 Ciccolini J, Dahan L, Andre N et al. Cytidine deaminase residual activity in serum is a predictive marker of early severe toxicities in adults after gemcitabine-based chemotherapies. J. Clin. Oncol. 28(1), 160-165 (2010).

10 Ciccolini J, Mercier C, Dahan L, Andre N. Integrating pharmacogenetics into gemcitabine dosing - time for a change? Nat. Rev. Clin. Oncol. 8(7), 439-444 (2011).

11 Raynal C, Ciccolini J, Mercier C et al. High-resolution melting analysis of sequence variations in the cytidine deaminase gene $(C D A)$ in patients with cancer treated with gemcitabine. Ther. Drug Monit. 32(1), 53-60 (2010).
12 Tibaldi C, Giovannetti E, Vasile E et al. Correlation of $C D A, E R C C 1$, and XPD polymorphisms with response and survival in gemcitabine/cisplatin-treated advanced nonsmall cell lung cancer patients. Clin. Cancer Res. 14(6), 1797-1803 (2008).

13 Dahan L, Ciccolini J, Mercier C et al. Treatment failure upon gemcitabine intake. Is cytidine deaminase extensivity the ideal culprit? J. Clin. Oncol. 28(Suppl.) Abstract e14558 (2010).

14 Mahlknecht U, Dransfeld Cl, Bulut N et al. SNP analyses in cytarabine metabolizing enzymes in AML patients and their impact on treatment response and patient survival: identification of $C D A$ SNP C-451T as an independent prognostic parameter for survival. Leukemia 23(10), 1929-1932 (2009).

15 Abraham A, Varatharajan S, Abbas S et al. Cytidine deaminase genetic variants influence RNA expression and cytarabine cytotoxicity in acute myeloid leukemia. Pharmacogenomics 13(3), 269-282 (2012).

16 Ciccolini J, Evrard A, M'Batchi L et al. CDA deficiency as a possible culprit for lifethreatening toxicities after cytarabine plus 6-mercaptopurine therapy: pharmacogenetic investigations. Pharmacogenomics 13(4), 393-397 (2012). 
17 Serdjebi C, Ciccolini J, Evrard A et al. Severe toxicities in patients undergoing gemcitabine, ARA-C, capecitabine, or azacytidine treatments: is deregulated cytidine deaminase the bad guy. Cancer Res. 72 (8 Suppl. 1) Abstract 1869 (2012).

18 Mercier C, Dupuis C, Blesius A et al. Early severe toxicities after capecitabine intake: possible implication of a cytidine deaminase extensive metabolizer profile. Cancer Chemother. Pharmacol. 63(6), 1177-1180 (2009).

19 Caronia D, Martin M, Sastre J et al. A polymorphism in the cytidine deaminase promoter predicts severe capecitabine-induced hand-foot syndrome. Clin. Cancer Res. 17(7), 2006-2013 (2011).
20 Fitzgerald SM, Goyal RK, Osborne WR, Roy JD, Wilson JW, Ferrell RE. Identification of functional single nucleotide polymorphism haplotypes in the cytidine deaminase promoter. Hum. Genet. 119(3), 276-283 (2006). 